Research Article

\title{
Research on a Critical Hydraulic Gradient of Piping in Noncohesive Soils
}

\author{
Can-Hong Zhang $\mathbb{D}^{1,2,3}$ En-Yue Ji $\mathbb{D}^{2,4}$ and Bao-Tian Wang $\mathbb{D}^{5}$ \\ ${ }^{1}$ Nanjing Vocational University of Industry Technology, Nanjing 210023, China \\ ${ }^{2}$ Key Laboratory of Failure Mechanism and Safety Control Techniques of Earth-Rock Dam of the Ministry of Water Resources, \\ Nanjing, China \\ ${ }^{3}$ Key Laboratory of Hydraulic and Waterway Engineering of the Ministry of Education, Jiaotong University, Chongqing, China \\ ${ }^{4}$ Nanjing Hydraulic Research Institute, Nanjing 210024, China \\ ${ }^{5}$ Hohai University, Nanjing 210098, China
}

Correspondence should be addressed to Can-Hong Zhang; 2008zhangcanhong@163.com

Received 26 August 2021; Accepted 27 November 2021; Published 18 December 2021

Academic Editor: Paolo Castaldo

Copyright (c) 2021 Can-Hong Zhang et al. This is an open access article distributed under the Creative Commons Attribution License, which permits unrestricted use, distribution, and reproduction in any medium, provided the original work is properly cited.

\begin{abstract}
The critical hydraulic gradient of cohesive soil is an important condition for judging soil piping. For force analysis of movable particles in pore channels of soil, this study proposes to consider the influence of surrounding particles on the drag force of movable particles by water flow. According to the principle of relative motion, considering the interaction force between moving objects in still water, the value of the drag force of water flow that is affected by surrounding particles is calculated, to derive the method of the critical hydraulic gradient. This calculation method is suitable for the results of previous piping tests, and the method is accurate and concise.
\end{abstract}

\section{Introduction}

The problem of water leakage has always been a technical problem that has plagued underground operations of civil engineering, and it has therefore been widely studied $[1,2]$. Piping is a kind of water seepage deformation; when studying piping laws and models, determining the criteria for the occurrence of piping is the primary issue. Research on the discriminant theory of piping has gone through a process from simple practical experience to a combination of practical experience and mechanical analysis. Many scholars have proposed a variety of methods to determine piping on the basis of different theoretical foundations and experimental data. Liu [3], Wu [4], and Sha [5] all deduced the calculation of the critical hydraulic gradient on the basis of the balance principle of a soil particle's own weight, hydrostatic buoyancy, and seepage force formula. Qi and $\mathrm{He}$ [6] proposed that the cause of particle loss is that the drag force of the particle destroys the original static equilibrium condition of the particle, and the drag force of the water current is related to the pore flow velocity. Wang et al. [7] considered the effective stress and fine-grain stress reduction, and they obtained the formula for calculating the critical hydraulic gradient of sandy soil fine-grain initiation during the subduction process, according to the limit force balance state. Wu et al. [8] proposed a method to initiate the critical slope of vertical upward infiltration via an unstable internal gravel soil particle group. The traditional piping identification method has certain limitations. It can be used to simply analyze the force of a single particle, and it does not consider the impact of the surrounding particles on the force of the particle when piping occurs. Piping is a process in which particles transform from being static to being in motion. The driving force of particle motion comes from the drag force of fluid. The interaction between solid particles affects the drag force. In this study, when analyzing the critical state of a static equilibrium failure of movable particles, the interaction between solid particles during soil 
piping is considered to derive the critical hydraulic gradient of noncohesive soil piping. Finally, in view of the particles' vertical movement, the formula is applied to the results of a one-dimensional piping test that was conducted by predecessors for verification.

\section{Physical Model of Particle Pore Channels}

Generally, noncohesive piping soil consists of the following two types of soil particles: framework particles and movable particles. The pore size of soil can be described by two capillary models that have different cross-sectional sizes [9], and the movement of movable particles in pores can be approximated as the movement of particles in a circular pipe fluid. The minimum diameter $\left(d_{0}\right)$ and maximum diameter $\left(d_{2}\right)$ of a pore channel are, respectively [9-11]:

$$
\begin{aligned}
& d_{0}=\frac{1}{\beta} \frac{8 n}{3(1-n)} D_{h}, \\
& d_{2}=1.86 d_{0},
\end{aligned}
$$

where $n$ is the porosity of the soil, $D_{h}$ is the effective particle size of the soil particles, and $\beta$ is the shape coefficient of the soil particles; the value is 6 for spherical particles.

Assuming that soil particles have the same shape, the effective diameter of soil particles can be obtained by the following formula $[12,13]$ :

$$
D_{h}=\frac{1}{\sum \Delta S_{i} / D_{i}},
$$

where $\Delta S_{i}$ is the mass percentage of the $i$ th particle group in the soil particles and $D_{i}$ is the representative particle size of the $i$ th particle group in the soil particles.

It is assumed that when the particle size is less than or equal to the smallest diameter $\left(d_{0}\right)$ of the pore channel, the soil is potentially unstable. According to the Poiseuille circular tube laminar flow theory [14-16], the water flow and velocity in the pore channel in the pore model are as follows:

$$
\begin{aligned}
& Q=\pi\left(\frac{\gamma_{w}}{\mu_{w}}\right)\left(\frac{d_{0}^{4}}{128}\right) J, \\
& v=\left(\frac{\gamma_{w}}{\mu_{w}}\right)\left(\frac{d_{0}^{2}}{32}\right) J,
\end{aligned}
$$

twhere $\gamma_{w}$ is the weight of water, $\mu_{w}$ is the viscosity of water, $d_{0}$ is the smallest diameter of the pore channel of soil particles, and $J$ is the hydraulic gradient at both ends of the channel.

\section{Force Analysis of Particles in Pore Channels}

When the drag force of a water flow on fine particles in a pore channel destroys the original static equilibrium state of the fine particles, they move along the pore channel under the drag of the water flow; ultimately, they are taken to the surface. Therefore, the essence of piping is the process in which the balance of the forces of particles is destroyed and then taken out.

The forces on a movable particle in the flow field are shown in Figure 1. The forces that the movable particle

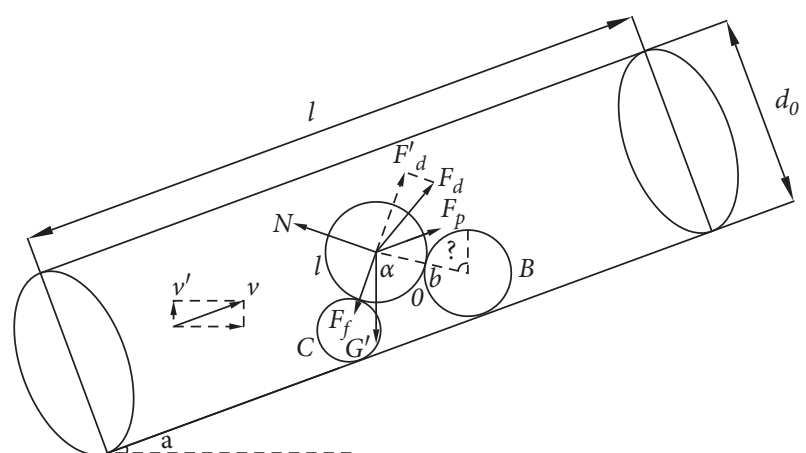

FIGURE 1: Schematic diagram of the forces operating on particle in pores.

experiences before it moves are the drag force $\left(F_{d}\right)$ of the seepage water flow, underwater gravity $\left(G^{\prime}\right)$, the supporting force $(N)$ of surrounding particles, the hydrostatic pressure on the surface $\left(F_{p}\right)$, and the friction between particles $\left(F_{f}\right)$. The direction of the hydrostatic pressure on the surface of the particles is consistent with the direction of the pore flow velocity. The drag force that is experienced by the particles is related to the pore flow velocity. When the pore velocity is greater, the drag force experienced by the particles is greater. However, the drag force is also affected by the surrounding particles, and thus, the direction of the drag force and that of the pore flow velocity are not necessarily the same.

The drag force gradually increases with an increase in the pore flow rate. When the total moment of the forces on a movable particle around the tangent point $\mathrm{O}$ is zero, the static balance of the movable particle reaches the limit state. At this point, the pore flow velocity in the pore channel is the critical flow velocity for particle initiation; this is also called the initiation velocity. When the particles move, the movable particles roll along the surface of the surrounding particles, and the frictional force that is generated is a rolling frictional force between the two particles. Generally, the rolling friction is relatively small, and thus, it can be ignored in the calculation. When a particle moves, the upstream particle $\mathrm{C}$ separates from particle $\mathrm{A}$, and the supporting force and frictional force of particle $\mathrm{C}$ with respect to particle $\mathrm{A}$ are zero. Therefore, when analyzing the force of particle $\mathrm{A}$, the interaction between particle A and particle B is mainly considered.

On the basis of the above principle, when particle A starts, the force acting on particle A reaches a momentary balance. For the tangent point $\mathrm{O}$, the following is true:

$$
F_{d}^{\prime}+F_{p} \cos (\theta-\alpha)-G^{\prime} \sin \theta=0,
$$

where $F_{d}^{\prime}$ is the drag force of the particles in the vertical direction along the line between the centers of the two particles, $F_{p}$ is the hydrostatic pressure acting on the surface of the particles, $G^{\prime}$ is the floating weight of the particles, $\theta$ is the angle between the line between the centers of the two particles and the vertical direction, and $\alpha$ is the angle between the direction of water flow and the horizontal plane.

The drag force of a water flow that is affected by surrounding particles can be obtained by the interaction 
between two moving objects in still water. When the two balls $\mathrm{A}$ and $\mathrm{B}$ move in a direction that is perpendicular to the line that connects their centers in still water, the radii of the two balls are, respectively, a and $b$, and the moving speeds are, respectively, $v_{a}$ and $v_{b}$; then, the resistance acting on ball A [16] can be expressed as follows:

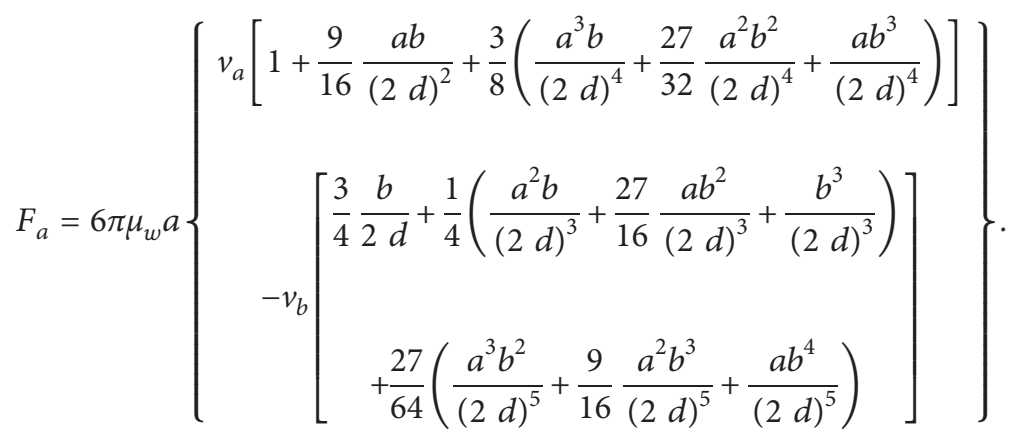

In this formula, $\mu_{w}$ is the viscosity coefficient of water and $2 d$ is the distance between the centers of two particles. When the two particles are in contact, then the following is true: $2 d=a+b$.
To facilitate the writing and expression of formula (5), the parameter $\eta$ is used to simplify it. The following formula (6) is the concrete expression of the parameter $\eta$ :

$$
\eta=\left\{\begin{array}{c}
{\left[\begin{array}{c}
1+\frac{9}{16} \frac{a b}{(a+b)^{2}}+\frac{3}{8}\left(\frac{a^{3} b}{(a+b)^{4}}+\frac{27}{32} \frac{a^{2} b^{2}}{(a+b)^{4}}+\frac{a b^{3}}{(a+b)^{4}}\right) \\
-\left[\begin{array}{c}
\frac{3}{4} \frac{b}{a+b}+\frac{1}{4}\left(\frac{a^{2} b}{(a+b)^{3}}+\frac{27}{16} \frac{a b^{2}}{(a+b)^{3}}+\frac{b^{3}}{(a+b)^{3}}\right) \\
+\frac{27}{64}\left(\frac{a^{3} b^{2}}{(a+b)^{5}}+\frac{9}{16} \frac{a^{2} b^{3}}{(a+b)^{5}}+\frac{a b^{4}}{(a+b)^{5}}\right)
\end{array}\right]
\end{array}\right\} .} \\
F_{d}^{\prime}=6 \pi \mu_{w} a v \eta \cos (\theta-\alpha) .
\end{array}\right\}
$$

According to the principle of relative motion, the drag force $\left(F_{d}^{\prime}\right)$ of the water velocity component $(v \prime)$ along the vertical direction of the centerline on particle $A$ can be calculated using equation (5). The speed of the two balls moving in the vertical direction along the centerline in still water is determined by the principle of relative motion. This can be regarded as the water flow velocity along the vertical direction of the centerline between the two stationary particles. The resistance acting on ball $\mathrm{A}$ is the drag force of the water flow on particle $\mathrm{A}$, and the direction is the same as the component direction of the water flow velocity $(v /)$ :

$$
F_{d}^{\prime}=6 \pi \mu_{w} a v^{\prime} \eta
$$

where $v^{\prime}$ is the water flow velocity along the vertical direction of the centerline of the two particles. That is, the component of the pore flow velocity along the vertical direction of the centerline of the two particles is as follows:

$$
v^{\prime}=v \cos (\theta-\alpha) \text {. }
$$

Substituting equations (8) into (7) results in the following:
According to the resistance of the particles when the two particles move in the direction of the centerline in still water [14], the drag force of the water velocity component along the centerline of the two particles on particle A can also be obtained. According to the required drag force along the vertical direction of the centerline and the direction of the centerline, the size and direction of the total drag force of particle A can be obtained by considering the particle's interaction. In the analysis and calculation of the critical condition of particles in this study, the moment of the drag force along the centerline direction to the tangent point $\mathrm{O}$ is zero, and hence, it cannot be calculated.

The hydrostatic pressure $\left(F_{p}\right)$ can be given by the following formula [15]:

$$
F_{p}=J \gamma_{w} l \pi a^{2}
$$

where $\gamma_{w}$ is the weight of the water; $l$ is the length of the pore channel, which may be expressed as $l=2 a$ [12]; and $J$ is the hydraulic gradient at both ends of the channel. The 
relationship between the hydraulic gradient and the pore flow velocity can be obtained from equation (3):

$$
J=\frac{32 \mu_{w} v}{\gamma_{w} d_{0}^{2}}
$$

Substituting equations (11) into (10) and using $l=2 a$, the relationship between hydrostatic pressure and pore flow velocity can be obtained and expressed as follows:

$$
F_{p}=\frac{64 \mu_{w} v}{d_{0}^{2}} \pi a^{3}
$$

The floating weight expression of particles is as follows:

$$
G^{\prime}=\frac{4}{3} \pi a^{3}\left(\gamma_{s}-\gamma_{w}\right)
$$

where $\gamma_{s}$ is the weight of soil particles. By substituting formulae (9), (12), and (13) into (4), the critical pore flow rate is obtained after sorting. That is, the expression of the starting flow rate is as follows:

$$
v_{c r}=\frac{2 a^{2}\left(\gamma_{s}-\gamma_{w}\right) \sin \theta}{\left(9 \mu_{w} \eta+96 \mu_{w} a^{2} / d_{0}^{2}\right) \cos (\theta-\alpha)} .
$$

Combining equation (3) with the relationship between the hydraulic gradient and the pore flow velocity, the critical hydraulic gradient at the starting particle can be obtained as follows:

$$
J_{c r}=\frac{64 a^{2}\left(\gamma_{s}-\gamma_{w}\right) \sin \theta}{\left(9 \eta \gamma_{w} d_{0}^{2}+96 \gamma_{w} a^{2}\right) \cos (\theta-\alpha)},
$$

where $\theta$ is the angle between the centerline of the two particles and the vertical direction, which represents the position of the soil particles. Therefore, the standard for starting particles with radius a is obtained using equation (15) and takes into account the influence of factors such as particle radius, porosity, soil composition, location of soil particles, and direction of pore water flow. The influence of surrounding particles on the drag force of starting particles is also considered.

In a double-layer embankment, the occurrence of piping is marked by the formation of piping openings. These occur in weak places behind dams, such as in ponds and pumped wells. After piping opening forms, the water in the dam gushes out from the piping opening through a permeable layer, and fine particles in the permeable layer are removed from the soil under the action of water flow to form a piping. The movement of particles at the piping mouth is a bottomup process, and thus, the particles must be removed to overcome the restriction of the upward movement of particles. Therefore, at the piping mouth, particles are pushed by the vertical water flow when they move vertically upward; $\alpha=90^{\circ}$, which is the angle between the water flow direction and the horizontal plane. To simplify the calculation, it is assumed that the particle diameters of the movable particles are equal (i.e., $a=b$ ), and this is substituted into equation (6) to obtain $\eta=0.683$. Assuming that the two particles are on the same plane, $\theta=90^{\circ}$. The values of $\alpha, \theta, \eta$ are substituted into equation (15), and the simplified critical hydraulic gradient formula for the vertical starting of movable particles is obtained as follows:

$$
J_{c r}=\frac{G_{s}-1}{1.5+0.38\left(d_{0} / 2 a\right)^{2}}
$$

In this formula, $G_{s}$ is the specific gravity of soil particles, and its value is $G_{s}=\gamma_{s} / \gamma_{w}$. Formula (16) is similar to the well-known Kantlaev impermeability gradient formula [3], which can be expressed as follows:

$$
J_{c r}=\frac{G_{s}-1}{1+0.43\left(d_{0} / d_{B}\right)^{2}},
$$

where $d_{B}$ is the particle size of the movable particles.

In formula (16), $a$ is the radius of the movable particles and $2 a$ is the diameter of the movable particles. From comparing formulae (16) and (17), it can be found that formula (16) and Kantlaev's anti-permeability gradient formula have the same form, but the coefficients in the denominator of the formula are different. The formula for Kantlaev's impermeability ratio is also derived from the force balance conditions of soil particles, and thus, the two formulae have the same form. However, when calculating the force of the particles, this study takes into account the influence of the drag force of the water flow on the surrounding particles. The calculated water drag force is different from that obtained using Kantlaev's formula, and the coefficients in the two equations are different. The following section discussed the verification and comparison of the vertical critical hydraulic gradient formula (16) and Kantlaev's impermeability gradient formula (17) using the test data of Skempton [11] and others.

\section{Calculation Examples and Analysis}

The test data of Skempton et al. were taken from the literature [11], and the gradation curve of the sand and gravel material that was used in the test is shown in Figure 2. Skempton et al. prepared 4 types of sands (A, B, C, and D) with a height of $155 \mathrm{~mm}$. These were placed in a transparent tube that had an inner diameter of $139 \mathrm{~mm}$, which is convenient for observing the movement of fine particles on the sidewall of the tube. A filter layer was placed at the lower end of the round pipe, and the water flow direction was from bottom to top. A number of piezometer tubes were arranged along the height of the soil sample to measure the pore water head in the soil sample. During the whole test, the water temperature was kept at $20^{\circ} \mathrm{C}$, the particle gradation of soil sample A was discontinuous, and the particle gradation of soil samples B, C, and D was continuous. The test results show that piping occurred in soil samples $\mathrm{A}$ and $\mathrm{B}$; the critical hydraulic gradients were 0.20 and 0.34 , respectively.

According to the calculations of Liu and Miao [17], the boundary diameters between the skeleton particles and the movable particles in these two soil samples are $1.0 \mathrm{~mm}$ and $0.43 \mathrm{~mm}$ for soil samples $\mathrm{A}$ and $\mathrm{B}$, respectively, and the mass percentages of the movable particles are $15 \%$ and $8.72 \%$, respectively. However, not all of the movable particles that 


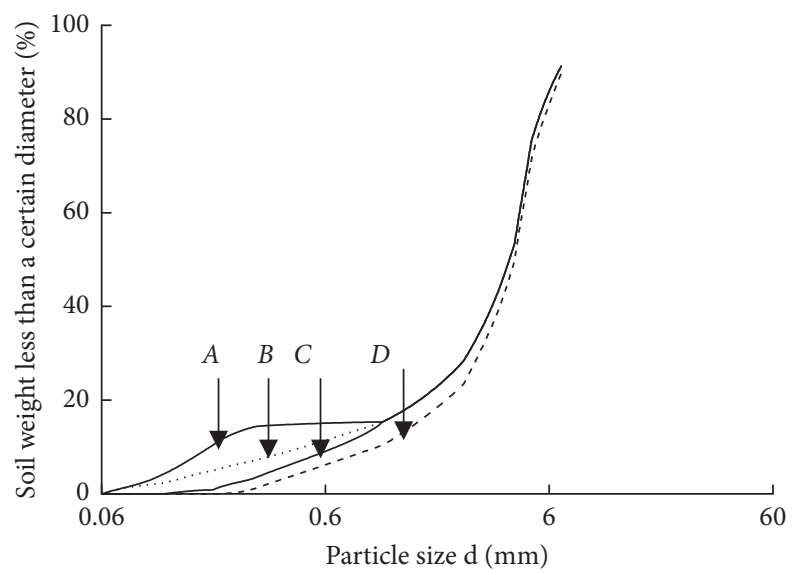

FIGURE 2: Grading curves of sandy gravel soil in Skempton's experiment.

TABLE 1: Soil sample parameters, test results, and calculation results.

\begin{tabular}{lccccccc}
\hline Soil sample & $n$ & $G_{\mathrm{s}}$ & $2 a(\mathrm{~mm})$ & $d_{0}(\mathrm{~mm})$ & Jcr test value & \multicolumn{2}{c}{ Jcr test value } \\
& & & & & Formula (16) & Kantlaev's formula \\
\hline A & 0.34 & 2.60 & 0.12 & 0.57 & 0.20 & 0.16 & 0.15 \\
B & 0.37 & 2.60 & 0.15 & 0.42 & 0.34 & 0.36 & 0.37 \\
\hline
\end{tabular}

have a particle size smaller than the particle size limit can be washed out of the soil. Here, we take the hydraulic gradient that corresponds to the loss of soil particles accounting for $3 \%$ of the total soil mass as the critical point when piping occurs. The hydraulic gradient (i.e., the particle size of the moving particles) is taken as $2 a=d_{3}$ (where $d_{3}$ is the soil particles smaller than the size accounts for $3 \%$ of the total mass of the soil). According to the gradation curve of soil samples A and B, the effective particle size $\left(D_{h}\right)$ of the soil particles can be obtained, and then, the porosity $(n)$ of the soil and the calculated value $\left(D_{h}\right)$ can be substituted into formula (1) to obtain the minimum diameter $\left(d_{0}\right)$ of the pore channel of the soil particles.

In the theoretical calculation of soil samples A and B, $G_{s}=2.60$, and the other parameters are shown in Table 1 . The hypothetical conditions met in the test and the parameters that were used were substituted into formulae (16) and (17) for the calculations. The calculation results are shown in Table 1 . The characteristics of soil materials, test results, and the calculation results of the formula are all listed in the table.

The calculated critical hydraulic gradient for piping of the soil sample is compared with the experimental value. This comparison shows that for soil sample A, the calculation result of formula (16) and the calculation result of Kantlaev's equation are slightly smaller than the experimental result reported in the literature [11]. The difference is within the acceptable range. The calculation result of formula (16) is closer to the test result, and it is in good agreement with the test result. For soil sample B, the calculation results of formula (16) and Kantlaev's formula are both consistent with the test results. According to the above calculation examples, the following can be concluded: (1) since the gradation of soil sample $B$ is more continuous than that of soil sample $A$, the movable particle size of soil sample $\mathrm{B}$ is closer to the smallest diameter of the pore channel. Thus, the calculation results for soil sample $B$ using the two theoretical formulae are more consistent. Test value: (2) the critical hydraulic gradient of particle initiation that is calculated by equation (16) is closer to reality. This is an improvement of Kantlaev's impermeability gradient formula, which can be used to calculate the critical hydraulic gradient of vertical particle initiation at a gushing mouth.

\section{Conclusions}

By analyzing the movement and force of the movable particles in the pore channel, the calculation formula of the critical hydraulic gradient for the particle movement is deduced. When analyzing the forces of particles, the drag force of the particles is affected by the surrounding particles. According to the scientific literature [14], when two balls move in still water, the formula for calculating the resistance that acts on one of the balls is given by the relative motion. This principle shows that the moving speed of the two balls in still water can be regarded as the water flow speed when the particles are at rest, and the resistance that acts on one of the balls is the drag force of the water flow on the particles. The derived formula that can be used to calculate the critical hydraulic gradient has the same form as the Kantlaev impermeability gradient formula. When calculating the force of the particles, the influence of the surrounding particles is taken into account; thus, the coefficients in the two formulae are different. Using previous test data [11], the judgment method was verified and compared with Kantlaev's impermeability ratio reduction formula. The critical hydraulic 
gradient that was calculated in this study is closer to the actual working conditions, and it is an improvement of Kantlaev's impermeability gradient ratio formula.

\section{Data Availability}

The data used to support the findings of this study are available from the corresponding author upon request.

\section{Conflicts of Interest}

The authors declare that they have no conflicts of interest.

\section{Acknowledgments}

This study was supported by the Key Laboratory of Hydraulic and Waterway Engineering of the Ministry of Education, Chongqing Jiaotong University (No. SLK2021A06), the Open Research Fund of Key Laboratory of Failure Mechanism and Safety Control Techniques of Earth-Rock Dam of the Ministry of Water Resources (No. YK321006), the Natural Science Research Project of Jiangsu Colleges and Universities (No. 19KJB560016), and the Talent Introduction Startup Fund Project of Nanjing Vocational University of Industry Technology (No. YK19-02-02).

\section{References}

[1] P. Castaldo, F. Jalayer, and B. Palazzo, "Probabilistic assessment of groundwater leakage in diaphragm wall joints for deep excavations," Tunnelling and Underground Space Technology, vol. 71, pp. 531-543, 2018.

[2] C. Moormann, "Analysis of wall and ground movements due to deep excavations in soft soil based on a new worldwide database," Soils and Foundations, vol. 44, no. 1, pp. 87-98, 2004.

[3] J. Liu, Seepage Stability and Seepage Control of Soil, China Water and Power Press, Beijing, China, 1992.

[4] L. J. Wu, "Calculation of critical hydraulic gradient non-cohesive soil," Journal of Nanjiang Hydraulic Research Institute, vol. 1, no. 4, pp. 90-95, 1980.

[5] J. X. Sha, "Research on piping in porous media," Journal of Nanjiang Hydraulic Research Institute, vol. 3, pp. 89-93, 1981.

[6] Q. W. Qi and M. M. He, Sediment Initiation Law and Initiation Velocity, China Science Publishing, Beijing, China, 1999.

[7] M. N. Wang, Y. T. Jiang, L. Yu, Y. C. Dong, and R. Y. Duan, "Analytical solution of startup critical hydraulic gradient of fine particles migration in sandy soil," Rock and Soil Mechanics, vol. 41, no. 8, pp. 2515-2524, 2020.

[8] M. X. Wu, G. Y. Gao, J. X. Yang, and Z. G. Zhan, "A method of predicting critical gradient for piping of sand and gravel soils," Rock and Soil Mechanics, vol. 40, no. 3, pp. 861-870, 2019.

[9] Z. Y. Liu, J. C. Le, and T. D. Miao, "Capillary-tube model for piping in noncohesive soils and its application," Chinese Journal of Rock Mechanics and Engineering, vol. 23, no. 22, pp. 3871-3876, 2004.

[10] Z. Y. Liu, "Critical hydraulic gradient for piping in noncohesive soils," Journal of Zhengzhou University (Philosophy and Social Sciences Edition), vol. 24, no. 4, pp. 67-71, 2003.

[11] A. W. Skempton and J. M. Brogan, "Experiments on piping in sandy gravels,” Géotechnique, vol. 44, no. 3, pp. 449-460, 1994.
[12] G. Kovacs, Seepage Hydraulics, Elsevier Scientific Publishing Company, New York, NY, USA, 1981.

[13] J. Happel, Low Reynolds Number Hydrodynamics, Noordhof, Leyden, IL, USA, 1973.

[14] C. S. P. Ojha, V. P. Singh, and D. D. Adrian, "Determination of critical head in soil piping," Journal of Hydraulic Engineering, vol. 129, no. 7, pp. 511-518, 2003.

[15] B. Indraratna and S. Radampola, "Analysis of critical hydraulic gradient for particle movement in filtration," Journal of Geotechnical and Geoenvironmental Engineering, vol. 128, no. 4, pp. 347-350, 2002.

[16] S. L. Wang, Fluid Mechanics, China Electric Power Press, Beijing, China, 2004.

[17] Z. Y. Liu and T. D. Miao, "Assessment for the noncohesive piping-typed soils," Rock and Soil Mechanics, vol. 25, no. 7, 2004. 